gesucht, daß das Ereignis während einer Beobachtungszeit $T$ wenigstens einmal mindestens $t$ Sekunden lang ausbleibt, daß also das längste ereignisfreie Zeitintervall $z$ wischen $T_{0}$ und $T_{0}+T$ mindestens die Länge $t$ besitzt. Das genaue Resultat ist:

$$
\begin{gathered}
w= \\
-x(T-2 t)\left(1+\frac{x(T-2 t)}{2}\right) e^{-2 x t} \\
+\frac{x^{2}}{2 !}(T-3 t)^{2}\left(1+\frac{x(T-3 t)}{3}\right) e^{-3 x t}-+\ldots
\end{gathered}
$$

wobei nur die Glieder zu nehmen sind, für welche $T \geqq n t$ ist $(n=1,2,3, \ldots)$.

Ist $T$ groß gegeniiber $t$, so gilt mit $\eta=x t e^{-x t}$ und $x=x T e^{-* t}$ in hoher Annäherung:

wobei

$$
w=1-\left(\varphi-e^{-x t} \psi\right) e^{-x},
$$

$$
\begin{aligned}
y & =1-\eta(x-1)+\frac{\eta^{2}}{2 !}\left(x^{2}-5 x+4\right)- \\
& -\frac{\eta^{3}}{3 !}\left(x^{3}-12 x^{2}+37 x-27\right)+\ldots \\
\psi & =1-\eta(x-2)+\frac{\eta^{2}}{2 !}\left(x^{2}-7 x+y\right)- \\
& -\frac{\eta^{3}}{3 !}\left(x^{3}-15 x^{2}+61 x-64\right)+\ldots
\end{aligned}
$$

ist.

Für kleine Werte von $\eta x$ erhält man die Näherungsformel:

$$
w=1-e^{-x T} e^{-x t} .
$$

Ist $z$. B. $t=5^{\mathrm{sec}}, T=3600^{\mathrm{sec}}$, so wird bei $x=1$ $w=1-\frac{1}{70000000000}$, während sich für $x=2$ und $x=3$ die Werte $w=0,279$ und $w=0,0033$ ergeben. Größere Pausen zufälliger Natur sind an sich seltene Erscheinungen, die aber bei längerer Beobachtungsdauer doch mit hoher Wahrscheinlichkeit auftreten muissen.

Ist $t$ die Länge des größten ereignisfreien Intervalls während der Beobachtungszeit $T$, so kann man, wenn $T$ grob gegenüber $t$ ist, nach der Formel

$$
x=\frac{0,159+\log T+\log x}{0,434 t},
$$

in welcher $\log$ den gemeinen Logarithmus bezeichnet, einen wahrscheinlichen Wert der Häufigkeit $x$ bestimmen. Dies gilt auch, wenn die Einzelereignisse, wie bei den Höhenstrahlen, von kurzen "Schauern" begleitet sind, die eine direkte Zählung stören können.

\section{P. FINSLER}

Mathematisches Institut der Universität, Zürich, den 14. April 1945.

\section{Uber die Quaternionenmultiplikation regulärer vierfachperiodischer Funktionen}

In einer frühern Arbeit ${ }^{1}$ habe ich alle vierfachperiodischen rechtsregulären Funktionen einer Quaternionenvariablen definiert und aufgestellt. Dabei blieb noch ein allgemeiner funktionentheoretischer Satz, der vermutungsweise ausgesprochen wurde, unbewiesen.

1 R. Fueter: Uber vierfachperiodische Funktionen, Monatshefte f. Math. u. Phys. 4\$, 161 (1939).
Dieser Satz ist unterdessen von Herrn WALTER NEF bewiesen worden ', so da $B$ die in jener Arbeit ausgesprochenen Resultate nun vollständig bewiesen sind. Sind $\omega_{k}(k=1,2,3,4)$ die vier Perioden, deren Komponenten eine von Null verschiedene Determinante bilden müssen, und wählt man sie als Quaternionen des maximalen Integritätsbereiches J einer definiten BRANDIschen Quaternionenalgebra ${ }^{2}$, so läßt sich die Theorie der komplexen Multiplikation der elliptischen Funktionen weitgehend auf den Bereich der vierfachperiodischen Funktionen übertragen. Ist nämlich $w=f(z)$ irgendeine der vierfachperiodischen rechtsregulären Funktionen mit den Perioden $\omega_{i}$ aus $J$, und sind $\mu$ und $\nu$ zwei Quaternionen jenes maximalen Integritätsbereiches $J$, so ist offenbar:

$$
W=f(\mu z \nu) \mu
$$

rechtsregulär als Funktion von $z$, vierfachperiodisch und hat auBerdem die Perioden von $w=f(z)$. Deshalb kann $W$ durch die von mir eingeführten Funktionen $P_{n_{1} n_{2} n_{3}}\left(z ; \omega_{1}, \omega_{2}, \omega_{3}, \omega_{4}\right)$ dargestellt werden, so wie es in meiner zitierten Arbeit ${ }^{3}$ angegeben wurde. Man erhält so wichtige Funktionalgleichungen.

Diese Darstellung wird besonders einfach und elegant, wenn man als Funktion $f(z)^{*}$ eine der regulären vierfachperiodischen Funktionen $p_{n_{1} n_{2} n_{3}}(z)$ selbst wählt. $p_{n_{1} n_{2} n_{3}}(\mu z \nu) \mu$ hat im Innern des Parallelotopes der Perioden nur die isolierten unwesentlichen singulären Punkte:

$$
z=\mu^{-1} \lambda v^{-1}
$$

wo die $\lambda$ ein bestimmtes endliches System von (mod. $\mu, v$ ) inkongruenten Quaternionen von $J$ durchläuft. Letzteres heißt, daß für zwei verschiedene der $\lambda$, etwa $\lambda^{\prime}$ und $\lambda^{\prime \prime}$, das Quaternion $\mu^{-1}\left(\lambda^{\prime}-\lambda^{\prime \prime}\right) \nu^{-1}$ nicht in $J$ liegt. Zur vollen Entwicklung der Theorie müssen Sätze über die Nullstellen der vierfachperiodischen Funktionen aufgestellt werden, was bisher nicht geschehen ist.

Rud. Fueter

Mathematisches Institut der Universität, Zürich, den 15. April 1945.

1 W. Ner; Die whwesentichen Singularitäten der reguläten Funktionest eituer Quaternionemvariablen, Comm. Math. Helv, 16, 284 (1943) 44).

2 H. BRANDT: Idealtheorie in Qwaternionenalgebren, Math. Ann.99, 1 (1928). Siehe auch dic beiden Arbeiten: R. FueTER: Quaternionenringe, Comm. Math. Helv, 6, 199 (1933/34); R. Fueter: Zur Theorie der Brandischen Quaternionenalgebren, Math. Ann. 110,650 (1985).

a R. FUETER: Uber vierfachperiodische Funktionen, Monatshefte f. Math. u. Phys. 48, 161 (1939).

\section{Zur Gharakterisierung zellteilungswirksamer Substanzen an der Gewebekultur}

Zellteilungsgifte beeinflussen die Lebensvorgänge der ruhenden Zelle in weitem Konzentrationsbereich wenig. Sie lassen der Zelle die Fähigkeit, in das Teilungsstadium zu treten. Daraus resultiert eine veränderte Reaktionsbereitschaft, die erst den Effekt "zellteilungswirksamer " Stoffe ermöglicht. Verschiedene Autoren haben versucht, den Begriff der Zellteilungsgifte auf Grund morphologisch feststellbarer Wirkungsunterschiede $z u$ differenzieren (MoeliendorfF, Dustin, Bucher, Bujard u. a.),

Bei der Untersuchung dor Zellteilungswirkung einer Reihe chemisch verschiedener Stoffgruppen sind wir zur 